\title{
Is Chemotherapy Related with Erectile Dysfunction in Non-Urologic Cancer Patients?
}

\author{
Farzad Allameh ${ }^{1}$, Samira Azghandi ${ }^{2}$ and Morteza Fallah Karkan ${ }^{3, *}$ \\ ${ }^{1}$ MD-MPH, Laser Application in Medical Science Research Center, Shahid Beheshti University of Medical Sciences, Tehran, Iran \\ ${ }^{2}$ Radiation-Oncology Department, Shohada-e-Tajrish Hospital, Shahid Beheshti University of Medical Sciences, Tehran, Iran \\ ${ }^{3}$ Department of Urology, Shahid Beheshti University of Medical Sciences, Tehran, Iran \\ "Corresponding author: Department of Urology, Shahid Beheshti University of Medical Sciences, P.O. Box: 2649890691, Tehran, Iran. Email: mortezafallah.md@gmail.com.
}

Received 2018 July 23; Revised 2018 August 06; Accepted 2018 August 08.

\section{Dear Editor,}

Erectile dysfunction (ED) is characterized as men's inability to terminate or hold penile erection to complete the coitus (1). Sexual dysfunction (SD) contributes to quality of life (2). Malignancy can cause sexual adverse effects by direct and indirect ways. It can involve sexual organs, indirectly affect body image, or cause depression or fatigue with effects on libido (3). Most sexual disabilities are not caused by the cancer itself, but by side effects of cancer management (4). Chemotherapy and radiotherapy-related SD generally ends after the completion of therapeutic manipulations, whereas anatomical defects due to surgery might have a permanent effect on the patients' sexual activity $(2,5)$. Other aspects that play a role include subjects' age, general and urogenital co-morbidity, and degree of ED before cancer treatment (6-9).

Determining the incidence of $\mathrm{ED}$ in various chemotherapeutic regimens in these patients can lead to finding ways to improve quality of life, decrease anxiety, and increase acceptance in these patients.

In this prospective analytical study, 61 patients were treated with different chemotherapy regimens for nongenitourinary and central nervous system cancers, accepted written consent, and completed medical history; the patients were evaluated with the International index of erectile function (IIEF) questionnaire. Morning testosterone levels are also measured in the serum of patients. At the end of the month 3, after the start of chemotherapy, a reevaluation will be taken and the results will be analyzed.

After institutional review board approval was obtained, 61 patients with the mean age of $58.66 \pm 15.11$ years (range 30 - 81 years) signed written informed consent and were included in the study; two patients were excluded from the study due to a change in the treatment plan. The mean of testosterone concentration in the morning before and 3 months after the chemotherapy was $3.13 \pm 2.007$ and $3.30 \pm 2.19$, respectively, which was not statistically significant. The mean score of IIEF questionnaire before and 3 months after the chemotherapy was $11.29 \pm 2.42$ and $10.95 \pm 2.31$, respectively, which was not statistically significant (Table 1). In subgroup analysis, the mean score of the IIEF questionnaire in the group under treatment with Cisplatin-based chemotherapy drugs was used in patients with esophageal cancer, nasopharyngeal and mandibular cancer, and significantly decreased after treatment (before chemotherapy $11.53 \pm 2.03$ and, then, $10.27 \pm 2.57$ with $\mathrm{P}$ value: 0.02 ).

Sexual problems are nearly always caused by a combination of psychological parameters. Generally, having a diagnosis of cancer is regarded as a critical point in life associated with long-lasting psychological effects. Men may still suffer from anxiety, depression, and decreased sexual arousal, even a decade after neoplasm treatment (10).

The present study showed that patients treated with platinum-based chemotherapy agent had a sexual dysfunction, which could be due to the effect of this drug on germ cells and hypogonadism. Cancer treatment has multiple and variable side effects. Sexual abnormalities may be underestimated by physicians due to the use of various surgical procedures and may reduce the quality of life of patients. All patients should be informed of the treatment complications and choose the best treatment for cancer. 


\begin{tabular}{|c|c|c|c|}
\hline \multirow{2}{*}{ Chemotherapy Regimen } & \multicolumn{2}{|c|}{ Mean IIEF Score } & \multirow{2}{*}{ PValue } \\
\hline & Before Chemotherapy & After Chemotherapy & \\
\hline 5FU based ${ }^{\mathrm{a}}$ & $11.62 \pm 2.02$ & $11.46 \pm 1.19$ & 0.65 \\
\hline Carboplatine-based & $10.68 \pm 3$ & $10.58 \pm 2.83$ & 0.72 \\
\hline Cisplatine-based & $11.53 \pm 2.03$ & $10.27 \pm 2.57$ & 0.02 \\
\hline Cyclophosmamide-based & $11.56 \pm 2.69$ & $11.67 \pm 2$ & 0.76 \\
\hline Others & $11.67 \pm 57$ & $12.33 \pm 57$ & 0.18 \\
\hline Sum & $11.29 \pm 2.42$ & $10.95 \pm 2.31$ & 0.08 \\
\hline
\end{tabular}

a 5U: 5 Fluorouracil.

\section{Acknowledgments}

We are indebted to all the radiation-oncology ward staff

\section{Footnotes}

Authors' Contribution: None Declared.

Conflict of Interests: There was no conflict of interest.

Financial Disclosure: None.

\section{References}

1. Rahbar Taramsari M, Badsar A, Falahatkar S, Movassagh H, Fallah KarKan M, Montazeri S, et al. [The possible effects of methadone maintenance therapy on erectile dysfunction in male addicts visiting MMT centers of Rasht]. Iran J Toxicol. 2014;8(25):1081-5. Persian.

2. Cakar B, Karaca B, Uslu R. Sexual dysfunction in cancer patients: A review.J BUON. 2013;18(4):818-23. [PubMed: 24344003].

3. Voznesensky M, Annam K, Kreder KJ. Understanding and managing erectile dysfunction in patients treated for cancer. J Oncol Pract. 2016;12(4):297-304. doi:10.1200/JOP.2016.010678. [PubMed: 27072383]. [PubMed Central: PMC5015452].

4. Schover LR, van der Kaaij M, van Dorst E, Creutzberg C, Huyghe E, Kiserud CE. Sexual dysfunction and infertility as late effects of cancer treatment. EJC Suppl. 2014;12(1):41-53. doi: 10.1016/j.ejcsup.2014.03.004. [PubMed: 26217165]. [PubMed Central: PMC 4250536].

5. Yousefi Kashi AS, Khaledi S, Houshyari1 M. CT simulation to evaluate of pelvic lymph node coverage in conventional radiotherapy fields based on bone and vessels landmarks in prostate cancer patients. Iran J Cancer Prev. 2016;9(3). e6233. doi: 10.17795/ijcp-6233. [PubMed: 27703649]. [PubMed Central: PMC5038838].

6. Alemozaffar M, Regan MM, Cooperberg MR, Wei JT, Michalski JM, Sandler HM, et al. Prediction of erectile function following treatment for prostate cancer.JAMA.2011;306(11):1205-14. doi: 10.1001/jama.2011.1333. [PubMed: 21934053]. [PubMed Central: PMC3831607].

7. Karami H, Hassanzadeh-Hadad A, Fallah-Karkan M. Comparing monotherapy with tadalafil or tamsulosin and their combination therapy in men with benign prostatic hyperplasia: A randomized clinical trial. Urol J. 2016;13(6):2920-6. [PubMed: 27928815].

8. Salavati A, Mehrsai A, Allameh F, Alizadeh F, Namdari F, Hosseinian $\mathrm{M}$, et al. Is serum uric acid level correlated with erectile dysfunction in coronary artery disease patients? Acta Med Iran. 2016;54(3):173-5. [PubMed: 27107521].

9. Mehrsai AR, Namdari F, Salavati A, Dehghani S, Allameh F, Pourmand G. Comparison of transdermal electromotive administration of verapamil and dexamethasone versus intra-lesional injection for Peyronie's disease. Andrology. 2013;1(1):129-32. doi: 10.1111/j.20472927.2012.00018.x. [PubMed: 23258641].

10. Monti M, Rosti G, De Giorgi U, Cavallari G, Severini G, Giovanis P, et al. Sexual functions after high-dose chemotherapy in survivors of germ cell tumors. Bone Marrow Transplant. 2003;32(9):933-9. doi: 10.1038/sj.bmt.1704256. [PubMed: 14561995]. 